PROCEEDINGS OF THE

AMERICAN MATHEMATICAL SOCIETY

Volume 135, Number 11, November 2007, Pages 3589-3598

S 0002-9939(07)08881-8

Article electronically published on June 22, 2007

\title{
UNIFORM ALGEBRA ISOMORPHISMS AND PERIPHERAL MULTIPLICATIVITY
}

\author{
AARON LUTTMAN AND THOMAS TONEV
}

(Communicated by N. Tomczak-Jaegermann)

\begin{abstract}
Let $\varphi: A \rightarrow B$ be a surjective operator between two uniform algebras with $\varphi(1)=1$. We show that if $\varphi$ satisfies the peripheral multiplicativity condition $\sigma_{\pi}(\varphi(f) \varphi(g))=\sigma_{\pi}(f g)$ for all $f, g \in A$, where $\sigma_{\pi}(f)$ is the peripheral spectrum of $f$, then $\varphi$ is an isometric algebra isomorphism from $A$ onto $B$. One of the consequences of this result is that any surjective, unital, and multiplicative operator that preserves the peripheral ranges of algebra elements is an isometric algebra isomorphism. We describe also the structure of general, not necessarily unital, surjective and peripherally multiplicative operators between uniform algebras.
\end{abstract}

An important question in Banach algebra theory, which still lacks a satisfactory answer, is to find criteria for an operator between two Banach algebras to be linear and multiplicative. For linear operators between semisimple algebras an answer is suggested by the theorem of Gleason-Kahane-Żelazko (e.g., 11]) in terms of spectra of algebra elements. A theorem by Kowalski and Słodkowski [6] considers alternative spectral conditions for not necessarily linear operators. N. V. Rao and A. K. Roy [9] have introduced an interesting spectral multiplicativity condition that contributes to the matter. In particular for unital, that is $\Phi(1)=1$, operators $\Phi$ they have proven the following:

If $A$ is a uniform algebra on $X$ and $\Phi: A \rightarrow A$ is a surjective unital operator such that

$$
\sigma(\Phi(f) \Phi(g))=\sigma(f g)
$$

for every $f, g \in A$, where $\sigma(f)$ is the spectrum of $f$, then $\Phi$ is an isometric algebra isomorphism.

In the case when $A=C(X)$ this result was proven previously by Molnár [8]. More recently, Hatori, Miura and Takagi [4] have replaced (1) by the weaker range multiplicativity condition

$$
\operatorname{Ran}(\Phi(f) \Phi(g))=\operatorname{Ran}(f g),
$$

where $\operatorname{Ran}(f)=f(X)$ is the range of $f$. N. V. Rao, T. Tonev and E. Toneva [10] (see also 2]) considered a spectral additivity condition related to the peripheral

Received by the editors November 23, 2005 and, in revised form, August 14, 2006.

2000 Mathematics Subject Classification. Primary 46J10, 46J20; Secondary 46H40.

Key words and phrases. Uniform algebra, peaking function, peak set, generalized peak point, Choquet boundary, Shilov boundary, homeomorphism, spectrum of an element, peripheral spectrum, peripheral range, peripherally multiplicative operator, algebra isomorphism.

(C)2007 American Mathematical Society 
spectra of algebra elements and gave alternative conditions for an operator to be an algebra isomorphism.

In this paper we show that a surjective and unital operator between two uniform algebras is an isometric algebra isomorphism if it satisfies a multiplicativity condition that is weaker than (10) and related to the peripheral spectra. As a corollary we obtain that any surjective, unital, and multiplicative operator that preserves the peripheral ranges of algebra elements is an isometric algebra isomorphism.

\section{Peripheral speCtra of Algebra elements}

Let $A$ be a commutative Banach algebra with maximal ideal space $\mathcal{M}_{A}$ and Shilov boundary $\partial A$. Recall that the peripheral spectrum, $\sigma_{\pi}(f)$, of an element $f \in A$ is the maximum modulus set of the spectrum of $f$, that is, $\sigma_{\pi}(f)=\{z \in$ $\left.\sigma(f):|z|=\max _{w \in \sigma(f)}|w|\right\}$ (see e.g. [2]). If $A$ is a uniform algebra on $X$, then $\sigma_{\pi}(f)=\sigma(f) \cap \mathbb{T}_{\|f\|}$, where $\mathbb{T}_{\|f\|}$ is the circle in $\mathbb{C}$ centered at 0 with radius $\|f\|$. The spectrum, $\sigma(f)$, of an element $f \in A$ and its range, $\operatorname{Ran}(f)$, are not equal in general. As the following lemma shows, however, the peripheral spectrum $\sigma_{\pi}(f)$ and peripheral range of $f$, that is, the set $\operatorname{Ran}_{\pi}(f)=f(X) \cap\{z \in \mathbb{C}:|z|=\|f\|\}=$ $f(X) \cap \mathbb{T}_{\|f\|}$, do, in fact, always coincide.

Lemma 1. Let $A$ be a uniform algebra on a compact Hausdorff space $X$. Then $\sigma_{\pi}(f)=\operatorname{Ran}_{\pi}(f)$ for all $f \in A$.

Proof. Clearly, $\operatorname{Ran}_{\pi}(f) \subset \sigma_{\pi}(f)$, since the Gelfand transform of $f, \widehat{f}$, is an isometry and $\operatorname{Ran}(f)=f(X) \subset \widehat{f}\left(\mathcal{M}_{A}\right)=\sigma(f)$. Recall that $b \sigma(f) \subset \widehat{f}(\partial A)$, where $b \sigma(f)$ is the topological boundary of $\sigma(f)$ (cf. 1, 7]). The converse inclusion follows from $\sigma_{\pi}(f)=b \sigma(f) \cap \mathbb{T}_{\|f\|} \subset f(\partial A) \cap \mathbb{T}_{\|f\|} \subset f(X) \cap \mathbb{T}_{\|f\|} \subset \operatorname{Ran}_{\pi}(f)$.

In the sequel the terms peripheral range and peripheral spectrum will be used interchangeably.

Theorem 1 (MAIN THeOREM). Let $A$ and $B$ be uniform algebras. If a surjective and unital operator $\Phi: A \rightarrow B$ satisfies the condition

or, equivalently,

$(* *) \quad \sigma_{\pi}(\Phi(f) \Phi(g))=\sigma_{\pi}(f g)$,

for all $f, g \in A$, then $\Phi$ is an isometric algebra isomorphism between $A$ and $B$.

Note that in this theorem and elsewhere in this paper we do not assume the linearity of $\Phi$, unless specifically mentioned. Operators that satisfy the equivalent conditions $(*)$ and $(* *)$ for all $f, g \in A$ will be called peripherally multiplicative operators. Note that while $\operatorname{Ran}_{\pi}(f)$ is often easier to determine, $\sigma_{\pi}(f)$ and (**) are more convenient for use in the proofs that follow.

Peripherally multiplicative operators are not necessarily range-multiplicative. Indeed, let $A(\mathbb{D})$ be the disk algebra of continuous functions on the closed unit disk in $\mathbb{C}$ that are analytic on the interior of the disk. Define $\Phi: A(\mathbb{D}) \rightarrow C(\overline{\mathbb{D}})$ by $(\Phi(f))(z)=f(z) \cdot|z|$. For any $z \in \mathbb{T}$ we have $(\Phi(f) \Phi(g))(z)=$ $(f(z) \cdot|z|)(g(z) \cdot|z|)=f(z) g(z)$. Since any function $f \in A(\mathbb{D})$ attains all of its maximal modulus values on $\mathbb{T}$, it follows that $\sigma_{\pi}(\Phi(f) \Phi(g))=\sigma_{\pi}(f g)$, i.e., $\Phi$ is peripherally multiplicative. However, $\Phi$ is not range-multiplicative, since, for 
instance, $\operatorname{Ran}\left(\Phi(1)^{2}\right)=\operatorname{Ran}\left(|z|^{2}\right)=\overline{\mathbb{D}} \neq 1=\operatorname{Ran}\left(1^{2}\right)$. While $\Phi$ is an isometry, it is neither surjective, nor unital, nor an algebra isomorphism.

Let $A$ be a uniform algebra on a compact Hausdorff space $X$. Recall that an element $h \in A$ is a peaking function of $A$ if $\|h\|=1$, and $|h(x)|<1$ whenever $h(x) \neq 1, x \in X$. In this case $P(h)=\{x \in X: h(x)=1\}=h^{-1}\{1\}$ is the peak set (or, peaking set) of $h$. Observe that $h \in A$ is a peaking function of $A$ if and only if $\sigma_{\pi}(h)=\{1\}$. If $K \subset X$ is such that $K=P(h)$ for some peaking function $h$, we say that $h$ peaks on $K$. A point $x \in X$ is a generalized peak point, or a $p$-point, of $A$, if it equals the intersection of a family of peak sets of $A$. Equivalently, $x$ is a generalized peak point of $A$ if for every neighborhood $V$ of $x$ there is a peaking function $h$ of $A$ with $x \in P(h) \subset V$. The set $\delta A$ of all generalized peak points of $A \subset C(X)$ is called the Choquet boundary, or strong boundary, of $A$. It is known that $\delta A$ is a boundary of $A$, and its closure coincides with the Shilov boundary $\partial A$ of $A$, i.e. $\overline{\delta A}=\partial A$. Recall that an $x \in X$ is a peak point of $A$ if there is an $h \in A$ with $h(x)=1$ such that $|h(x)|<1$ for any $y \in X \backslash\{x\}$. Unlike generalized peak points, the set of peak points of $A$ is not necessarily dense in $\partial A$ if $X$ is not metrizable.

Denote the set of all peaking functions of $A$ by $\mathcal{F}(A)$. For a fixed $x \in X$ denote by $\mathcal{F}_{x}(A)$ the set of all peaking functions of $A$ with $x \in P(h)$, i.e. with $h(x)=1$.

Lemma 2. Let $A$ be a uniform algebra on $X$, and let $f, g \in A$. If $\|f h\| \leq\|g h\|$ for all peaking functions $h \in \mathcal{F}(A)$, then $|f(x)| \leq|g(x)|$ on $\partial A$.

Proof. Assume that $\|f h\| \leq\|g h\|$ for every $h \in \mathcal{F}(A)$, but $\left|f\left(x_{0}\right)\right|>\left|g\left(x_{0}\right)\right|$ for some $x_{0} \in \partial A$. Without loss of generality we may assume that $x_{0} \in \delta A$. Choose a $\gamma>0$ such that $\left|g\left(x_{0}\right)\right|<\gamma<\left|f\left(x_{0}\right)\right|$, and an open neighborhood $V$ of $x_{0}$ in $X$ so that $|g(x)|<\gamma$ on $V$. Let $h \in \mathcal{F}_{x_{0}}(A)$ be a peaking function of $A$ with $P(h) \subset V$. By choosing a sufficiently high power of $h$, we can assume from the beginning that $|(g h)(x)|<\gamma$ for every $x \in X \backslash V$. Since this inequality obviously holds also on $V$, we deduce that $\|g h\|<\gamma$. Hence,

$$
\|g h\|<\gamma<\left|f\left(x_{0}\right) h\left(x_{0}\right)\right| \leq\|f h\|,
$$

which contradicts the hypothesis. Consequently, $|f(x)| \leq|g(x)|$ on $\partial A$.

Corollary 1. Let $f, g \in A$. If $\|f h\|=\|g h\|$ for all peaking functions $h$ of $A$, then $|f(x)|=|g(x)|$ on $\partial A$.

If, moreover, $\sigma_{\pi}(f h)=\sigma_{\pi}(g h)$ for all $h \in \mathcal{F}(A)$, then we have the following identification lemma.

Lemma 3 (Identification Lemma). If $f, g \in A$ satisfy the condition

$$
\sigma_{\pi}(f h)=\sigma_{\pi}(g h)
$$

for every peaking function $h \in A$, then $f=g$, i.e. $f(x)=g(x)$ for all $x \in X$.

Proof. Clearly, $\|f h\|=\|g h\|$, since $|z|=\|f\|$ for every $z \in \sigma_{\pi}(f)$. It follows from Corollary 11 that $|f(x)|=|g(x)|$ on $\partial A$. Let $x_{0} \in \delta A$. If $f\left(x_{0}\right)=0$, then $\left|g\left(x_{0}\right)\right|=\left|f\left(x_{0}\right)\right|=0$ implies that also $g\left(x_{0}\right)=0$. Therefore, we can assume without loss of generality that $f\left(x_{0}\right) \neq 0$. Choose an open neighborhood $V$ of $x_{0}$ in $X$, and a peaking function $k \in \mathcal{F}_{x_{0}}(A)$ with $P(k) \subset V$. Let $x_{V} \in P(k)$ be such that $\left|f\left(x_{V}\right)\right|=\max _{\xi \in P(k)}|f(\xi)|$. Bishop's Lemma for uniform algebras (cf. [1, Theorem 2.4.1]) implies that there is a peaking function $h$ of $A$ with the same peaking set as $k$, so that the function $f h$ attains its maximum modulus 
exclusively within $P(h)$. In fact, we can choose $h$ so that both functions $f h$ and $g h$ attain the maxima of their modulus exclusively within $P(h)$. Therefore, by (2), $f\left(x_{V}\right)=f\left(x_{V}\right) k\left(x_{V}\right)=f\left(x_{V}\right) h\left(x_{V}\right) \in \sigma_{\pi}(f h)=\sigma_{\pi}(g h)$. Hence, there is a $z_{V} \in X$ so that $f\left(x_{V}\right)=(g h)\left(z_{V}\right)$ and $\left|(g h)\left(z_{V}\right)\right|=\|g h\|=\max _{\xi \in X}|(g h)(\xi)|$. Every such $z_{V}$ belongs to $P(h)$ by the choice of $h$. Therefore, $f\left(x_{V}\right)=(g h)\left(z_{V}\right)=g\left(z_{V}\right)$. Since in every neighborhood $V$ of $x_{0}$ there are points $x_{V}$ and $z_{V}$ in $V$ such that $f\left(x_{V}\right)=g\left(z_{V}\right)$, then $f\left(x_{0}\right)=g\left(x_{0}\right)$ by the continuity of $f$ and $g$. Consequently, $f(x)=g(x)$ on $\delta A$, and therefore, $f=g$.

\section{Peripherally multiplicative operators}

Let $A$ and $B$ be uniform algebras on $X$ and $Y$, respectively.

Lemma 4. If an operator $\Phi: A \rightarrow B$ is peripherally multiplicative, then for all $f, g \in A$ the following hold:

(a) $\|\Phi(f) \Phi(g)\|=\|f g\|$, i.e. $\Phi$ is norm-multiplicative;

(b) $\|\Phi(f)\|=\|f\|$, i.e. $\Phi$ preserves the norms.

If, in addition, $\Phi$ is unital, then

(c) $\sigma_{\pi}(\Phi(f))=\sigma_{\pi}(f)$, i.e. $\Phi$ preserves the peripheral spectra;

(d) $\sigma_{\pi}(\Phi(f) \Phi(g))=\sigma_{\pi}(\Phi(f g))$.

Proof. Equality (a) follows directly from $(* *)$, since $|z|=\|f\|$ for every $z \in \sigma_{\pi}(f)$. In a uniform algebra $\left\|f^{2}\right\|=\|f\|^{2}$, and hence $\|f\|^{2}=\left\|f^{2}\right\|=\left\|\Phi(f)^{2}\right\|=\|\Phi(f)\|^{2}$ by (a), which implies that $\|\Phi(f)\|=\|f\|$, i.e. (b) holds. Letting $g=1$ in (**) implies $(c)$, while $(d)$ follows from $(c)$ and $(* *)$ by $\sigma_{\pi}(\Phi(f g))=\sigma_{\pi}(f g)=\sigma_{\pi}(\Phi(f) \Phi(g))$.

It is straightforward to see that if $\Phi$ is unital, then the peripheral multiplicativity property $(* *)$ is equivalent to both $(c)$ and $(d)$.

Proposition 1. Every peripherally multiplicative operator $\Phi: A \rightarrow B$ is injective.

Proof. If $\Phi(f)=\Phi(g)$ for some $f, g \in A$, then for any peaking function $h \in \mathcal{F}(A)$ we have $\Phi(f) \Phi(h)=\Phi(g) \Phi(h)$; thus $\sigma_{\pi}(\Phi(f) \Phi(h))=\sigma_{\pi}(\Phi(g) \Phi(h))$. Hence

$$
\sigma_{\pi}(f h)=\sigma_{\pi}(\Phi(f) \Phi(h))=\sigma_{\pi}(\Phi(g) \Phi(h))=\sigma_{\pi}(g h),
$$

where we have used twice the peripheral multiplicativity of $\Phi$. The Identification Lemma 3 now implies that $f=g$. Thus $\Phi$ is injective.

Lemma 5. If $\Phi: A \rightarrow B$ is a surjective and peripherally multiplicative operator, then $\Phi(1)^{2}=1$.

This result is due to O. Hatori [5]. The proof presented here is due to S. Lambert.

Proof. Lemma 4(c) yields $\{1\}=\sigma_{\pi}\left(1^{2}\right)=\sigma_{\pi}\left(\Phi(1)^{2}\right)$. Consequently, $\Phi(1)^{2}$ is a peaking function of $B$. Let $k$ be a peaking function of $B$ and $h=\Phi^{-1}(k)$. Then, invoking Lemma $4(\mathrm{a}),(\mathrm{b})$,

$$
\|1 \cdot k\|=\|k\|=\|\Phi(h)\|=\|h\|=\|1 \cdot h\|=\|\Phi(1) \cdot \Phi(h)\|=\|\Phi(1) \cdot k\| .
$$

Corollary 11 implies that $|\Phi(1)|=1$, and therefore, $\left|\Phi(1)^{2}\right|=1$ on $\partial B$. Hence $\Phi(1)^{2}=1$ on $\partial B$, since, as noted above, $\Phi(1)^{2}$ is a peaking function of $B$. Consequently, $\left.\Phi(1)^{2} \equiv 1\right]$

\footnotetext{
${ }^{1}$ Thanks are due to Scott Lambert for permitting the publication of this proof.
} 
Lemma 5 implies that $\Phi(1)$ does not take values other than -1 and 1 , it is an invertible element of $B$, and $\Phi(1)^{-1}=\Phi(1)$. If $Y$ is connected, then $\Phi(1) \equiv 1$ or $\Phi(1) \equiv-1$.

Lemma 6. If $\Phi: A \rightarrow B$ is a surjective operator that preserves the peripheral spectra of algebra elements, then $\Phi(\mathcal{F}(A))=\mathcal{F}(B)$.

Proof. The inclusion $\Phi(\mathcal{F}(A)) \subset \mathcal{F}(B)$ follows from the preservation by $\Phi$ of peripheral spectra of algebra elements. Given $k \in \mathcal{F}(B)$, there exists $h \in A$ with $k=\Phi(h)$, by the surjectivity of $\Phi$. Clearly, $h \in \mathcal{F}(A)$ since $\sigma_{\pi}(h)=\sigma_{\pi}(\Phi(h))=\sigma_{\pi}(k)=\{1\}$. Hence $k=\Phi(h) \in \Phi(\mathcal{F}(A))$, and therefore $\mathcal{F}(B) \subset \Phi(\mathcal{F}(A))$. Consequently, $\Phi(\mathcal{F}(A))=\mathcal{F}(B)$, as claimed.

Definition 1 ([2, 10]). An operator $\Phi: A \rightarrow B$ is called monotone increasing in modulus if $|f(x)| \leq|g(x)|$ on $\partial A$ implies $|(\Phi(f))(y)| \leq|(\Phi(g))(y)|$ on $\partial B$ for every $f, g \in A$.

Lemma 7. If a surjective operator $\Phi: A \rightarrow B$ is norm-multiplicative, i.e. if

$$
\|\Phi(f) \Phi(g)\|=\|f g\|
$$

for every $f, g \in A$, then it is monotone increasing in modulus.

Proof. If $|f(x)| \leq|g(x)|$ on $\partial A$, then clearly $\|f h\| \leq\|g h\|$ for any $h \in A$. Since $\Phi$ is surjective, for any $k \in \mathcal{F}(B)$ there is an $h \in A$ such that $k=\Phi(h)$. Applying (3) twice we obtain $\|\Phi(f) \cdot k\|=\|\Phi(f) \Phi(h)\|=\|f h\| \leq\|g h\|=\|\Phi(g) \Phi(h)\|=$ $\|\Phi(g) \cdot k\|$ for every $k \in \mathcal{F}(B)$. Now Lemma 2 implies that $|(\Phi(f))(y)| \leq|(\Phi(g))(y)|$ on $\partial B$.

To prove the Main Theorem we will show that under its assumptions the mapping $\tau: \delta A \rightarrow \delta B$ considered in [9] is well defined, and the equality $(\Phi(f))(\tau(x))=f(x)$ holds for all $f \in A$ and $x \in X$.

Lemma 8. If $\Phi: A \rightarrow B$ is a surjective and monotone increasing in modulus operator that preserves the peripheral spectra of algebra elements, then for every generalized peak point $x \in \delta A$ the set

$$
E_{x}=\bigcap_{h \in \mathcal{F}_{x}(A)} P(\Phi(h))
$$

is nonempty.

Proof. Let $x$ be a generalized peak point of $A$. We will show that the family $\left\{P(\Phi(h)): h \in \mathcal{F}_{x}(A)\right\}$ has the finite intersection property. If $h_{1}, h_{2}, \ldots, h_{n}$ belong to $\mathcal{F}_{x}(A)$, then the function $g=h_{1} \cdot h_{2} \cdots h_{n}$ also belongs to $\mathcal{F}_{x}(A)$. Since $\left|h_{j}(x)\right| \leq$ $1, j=1, \ldots, n$, we have $|g(\xi)|=\left|h_{1}(\xi)\right| \cdot\left|h_{2}(\xi)\right| \cdots\left|h_{n}(\xi)\right| \leq\left|h_{k}(\xi)\right|$ for every $\xi \in \partial A$ and any fixed $k=1, \ldots, n$. According to Lemma [6, $\Phi(g)$ and $\Phi\left(h_{k}\right)$ are peaking functions of $B$. Since $\Phi$ is monotone increasing in modulus, it follows that $|(\Phi(g))(\eta)| \leq\left|\left(\Phi\left(h_{k}\right)\right)(\eta)\right|$ for every $\eta \in \partial B$. Consequently, for every $y \in Y$ with $(\Phi(g))(y)=1$ we must have $\left|\left(\Phi\left(h_{k}\right)\right)(y)\right|=1$; thus $\left(\Phi\left(h_{k}\right)\right)(y)=1$, which implies $P(\Phi(g)) \subset P\left(\Phi\left(h_{k}\right)\right)$. Since this holds for every $k=1, \ldots, n$, we deduce that $P(\Phi(g)) \subset \bigcap_{k=1}^{n} P\left(\Phi\left(h_{k}\right)\right)$. Consequently, the family $\left\{P(\Phi(h)): h \in \mathcal{F}_{x}(A)\right\}$ has the finite intersection property, as claimed. Hence it has a nonempty intersection, since all of its members are closed subsets of the compact set $Y$. 
Lemma 9. If $\Phi: A \rightarrow B$ is a surjective, unital and peripherally multiplicative operator, then for any generalized peak point $x \in \delta A$ the set $E_{x}$ defined in (4) is a singleton that belongs to $\delta B$.

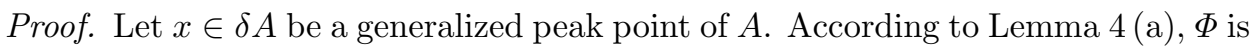
norm-multiplicative and, according to Lemma 7 monotone increasing in modulus. Since, in addition, $\Phi$ is unital, it preserves the peripheral ranges of algebra elements by Lemma $4(c)$. Thus $\Phi$ satisfies the hypotheses of Lemma 8 . Consequently, the family of peak sets $\left\{P(\Phi(f)): f \in \mathcal{F}_{x}(A)\right\}$ has a nonempty intersection, and therefore it meets $\delta B$ (cf. [7, Ex. 3,9, p.165]), i.e. $E_{x} \cap \delta B \neq \emptyset$.

Since $\Phi$ preserves the peripheral spectra of algebra elements, Lemma 6 yields $\Phi^{-1}(\mathcal{F}(B))=\mathcal{F}(A)$. We claim that

$$
\Phi^{-1}\left(\mathcal{F}_{y}(B)\right) \subset \mathcal{F}_{x}(A)
$$

for any $y \in E_{x} \cap \delta B$. Let $y_{0} \in E_{x} \cap \delta B, k \in \mathcal{F}_{y_{0}}(B)$, and let $h=\Phi^{-1}(k) \in \mathcal{F}(A)$. Since, by Proposition 1, $\Phi$ is injective, the function $h$ is uniquely defined. To show that $h \in \mathcal{F}_{x}(A)$ it is enough to see that $h(x)=1$. Take an open neighborhood $V$ of $x$ and a peaking function $g \in \mathcal{F}_{x}(A)$ with $P(g) \subset V$. By Lemma 6 we have $\Phi(g) \in \mathcal{F}(B)$. Since $g \in \mathcal{F}_{x}(A)$ and $y_{0} \in E_{x} \subset P(\Phi(g))$, we have $\Phi(g)\left(y_{0}\right)=1$; hence $\Phi(g) \in \mathcal{F}_{y_{0}}(B)$. Note that $k \cdot \Phi(g) \in \mathcal{F}_{y_{0}}(B)$. Lemma $4(a)$ yields $1=$ $\|k \cdot \Phi(g)\|=\|\Phi(h) \Phi(g)\|=\|h g\| \leq 1$. Hence $\|h g\|=1$, and there exists $x_{V} \in \partial A$ with $h\left(x_{V}\right)=g\left(x_{V}\right)=1$. Therefore, $x_{V} \in P(g) \subset V$. We deduce that any neighborhood $V$ of $x$ contains a point $x_{V}$ with $h\left(x_{V}\right)=1$. The continuity of $h$ implies that $h(x)=1$, so $h \in \mathcal{F}_{x}(A)$. Consequently, $\Phi^{-1}\left(\mathcal{F}_{y}(B)\right) \subset \mathcal{F}_{x}(A)$, as claimed.

Let $y_{0} \in E_{x} \cap \delta B$, and suppose that there exists a $y \in E_{x} \backslash\left\{y_{0}\right\}$. Then there is a peaking function $k \in \mathcal{F}_{y_{0}}(B)$ with $|k(y)|<1$. By (5), if $h=\Phi^{-1}(k)$, then $h \in \mathcal{F}_{x}(A)$. Hence $E_{x} \subset P(\Phi(h))=P(k)$, which implies $k(y)=1$, contradicting $|k(y)|<1$. This shows that the set $E_{x}$ contains exactly one point.

Let $\Phi: A \rightarrow B$ be a surjective, unital, and peripherally multiplicative operator, and let $x \in \delta A$. If $\tau(x)$ is the single element of the set $E_{x}$, i.e.,

$$
\{\tau(x)\}=E_{x}=\bigcap_{f \in \mathcal{F}_{x}(A)} P(\Phi(f)),
$$

then, as in [9], we can consider the mapping $\tau: x \longmapsto \tau(x)$ from $\delta A$ into $\delta B$. If $h \in \mathcal{F}_{x}(A)$, then, due to (4),$\tau(x) \in P(\Phi(h))$. Thus $(\Phi(h))(\tau(x))=1=h(x)$, and the equality

$$
(\Phi(h))(\tau(x))=h(x)
$$

holds for every peaking function $h \in \mathcal{F}_{x}(A)$. In the next section it will be shown that, under rather general conditions, equality (7) in fact holds for every $f \in A$ and any $x \in \delta A$.

Let $\Phi$ be a unital surjective and peripherally multiplicative operator. Let $k \in \mathcal{F}_{\tau(x)}(B)$ for some $x \in \delta A$, and let $\Phi^{-1}(k)=h \in \mathcal{F}(A)$. According to (5D), $\Phi^{-1}\left(\mathcal{F}_{\tau(x)}(B)\right) \subset \mathcal{F}_{x}(A)$; hence $h \in \mathcal{F}_{x}(A)$ and by (7) we have $k(\tau(x))=$ $(\Phi(h))(\tau(x))=h(x)=\left(\Phi^{-1}(k)\right)(x)$. Therefore, for every $x \in \delta A$ and any peaking function $k \in \mathcal{F}_{\tau(x)}(B)$ we have

$$
\left(\Phi^{-1}(k)\right)(x)=k(\tau(x)) .
$$


Lemma 10. Let $\Phi: A \rightarrow B$ be a surjective, unital, and peripherally multiplicative operator, and let $f \in A$. If $(\Phi(f))\left(\tau\left(x_{0}\right)\right)=0$ for some $x_{0} \in \delta A$, then also $f\left(x_{0}\right)=0$.

Proof. Let $x_{0}$ be a generalized peak point of $A$ and let $f \in A$. Choose an open neighborhood $U$ of $\tau\left(x_{0}\right)$ in $Y$, such that $|(\Phi(f))(y)|<\varepsilon$ on $U$. Let $k \in \mathcal{F}_{\tau\left(x_{0}\right)}(B)$ be a peaking function of $B$ with $P(k) \subset U$. By taking a high enough power of $k$, we may assume from the beginning that $|(\Phi(f))(y) \cdot k(y)|<\max _{\eta \in U}|(\Phi(f))(\eta) \cdot k(\eta)|<\varepsilon$ for all $y \in Y \backslash U$. Consequently, $\|\Phi(f) \cdot k\|<\varepsilon$, and according to Lemma $4(a)$, $\left\|f \cdot \Phi^{-1}(k)\right\|=\|\Phi(f) \cdot k\|<\varepsilon$. Hence by (8) we have $\left|f\left(x_{0}\right)\right|=\left|f\left(x_{0}\right) k\left(\tau\left(x_{0}\right)\right)\right|=$ $\left|f\left(x_{0}\right) \cdot\left(\Phi^{-1}(k)\right)\left(x_{0}\right)\right|<\varepsilon$. Thus, $\left|f\left(x_{0}\right)\right|<\varepsilon$, and consequently, $f\left(x_{0}\right)=0$ by the liberty of choice of $\varepsilon$.

Lemma 11. If $\Phi: A \rightarrow B$ is a surjective, unital, and peripherally multiplicative operator, then $|f(x)| \leq|(\Phi(f))(\tau(x))|$ for every $x \in \delta A$ and all $f \in A$.

Proof. Since $\Phi$ is peripherally multiplicative, then, according to Lemma $4(a),(b)$, $\|\Phi(f) \Phi(g)\|=\|f g\|$ and $\|\Phi(f)\|=\|f\|$ for all $f, g \in A$. Let $x \in \delta A, f \in A$, and let $g=\Phi(f) \in B$. Without loss of generality we can assume that $g(\tau(x)) \neq 0$, since, if $g(\tau(x))=(\Phi(f))(\tau(x))=0$, then, by Lemma 10, also $f(x)=0$, and the result carries. If $U$ is an open neighborhood of $\tau(x)$ in $Y$, then Bishop's Lemma allows us to choose a peaking function $k \in \mathcal{F}_{\tau(x)}(B)$ with $P(k) \subset U$, such that the function $(g k)(y)$ attains its maximum modulus within $P(k) \subset U$ exclusively. Let $\eta_{U} \in P(k)$ be such that $\left|g\left(\eta_{U}\right)\right|=\max _{\eta \in P(k)}|g(\eta)|$, and denote $h=\Phi^{-1}(k) \in \mathcal{F}_{x}(A)$. The norm-multiplicativity of $\Phi$ implies that

$$
\begin{aligned}
& |f(x)|=\left|f(x)\left(\Phi^{-1}(k)\right)(x)\right|=\left|\left(f \cdot \Phi^{-1}(k)\right)(x)\right| \\
& \leq\left\|f \cdot \Phi^{-1}(k)\right\|=\|\Phi(f) \cdot k\|=\|g k\|=\left|g\left(\eta_{U}\right)\right| .
\end{aligned}
$$

We have obtained that any neighborhood $U$ of $\tau(x)$ contains a point $\eta_{U}$ such that $|f(x)| \leq\left|g\left(\eta_{U}\right)\right|$. The continuity of $g$ implies that $|f(x)| \leq|g(\tau(x))|=$ $|(\Phi(f))(\tau(x))|$.

Lemma 12. If $\Phi: A \rightarrow B$ is a surjective, unital, and peripherally multiplicative operator, then the mapping $\tau$ from (6) is a homeomorphism from $\delta A$ onto $\delta B$.

Proof. According to Proposition 1, the operator $\Phi$ is bijective. Since the peripheral multiplicativity property (**) is symmetric with respect to $f$ and $\Phi(f)$, it holds also for the inverse operator $\Phi^{-1}$. According to Lemma 9, there arises a corresponding map $\psi: \delta B \rightarrow \delta A$ such that the equality (7), which in this case reduces to $\left(\Phi^{-1}(k)\right)(\psi(\eta))=k(\eta)$, holds on $\delta B$ for any $k \in \mathcal{F}_{\eta}(B)$. Let $x \in \delta A$ and $y=\tau(x) \in \delta B$. Then $h(\psi(y))=\left(\Phi^{-1}(k)\right)(\psi(y))=k(y)=(\Phi(h))(y)=$ $(\Phi(h))(\tau(x))=h(x)=1$, and therefore $\psi(y) \in P(h)$. Since this holds for every $h \in \mathcal{F}_{x}(A)$ and $\bigcap_{h \in \mathcal{F}_{x}(A)} P(h)=\{x\}$, we deduce that $\psi(y)=x$; thus $\psi(\tau(x))=x$ for every $x \in \delta A$. By similar arguments one can see also that $\tau(\psi(y))=y$ for any $y \in \delta B$. Consequently, $\tau$ and $\psi$ are injective mappings, and $\psi=\tau^{-1}$.

Let $x \in \delta A$ be a generalized peak point of $A$, and let $r \in(0,1)$. Choose an open neighborhood $V$ of $\tau(x)$ in $\delta B$, and a peaking function $k \in \mathcal{F}_{\tau(x)}(B)$ with $P(k) \subset V$ and $|k(y)|<r$ on $\delta B \backslash V$. If $h=\Phi^{-1}(k)$, then $h \in \mathcal{F}_{x}(A)$. Note that since $h(x)=1>r$, the open set $W=\{\xi \in \delta A:|h(\xi)|>r\}$ contains $x$. According to Lemma 11, for every $\xi \in W$ we have $|k(\tau(\xi))|=|(\Phi(h))(\tau(\xi))| \geq|h(\xi)|>r$, and 
therefore, $\tau(\xi) \in V$, since on $\delta B \backslash V$ we have $|k(\eta)|<r$. Consequently, $\tau(W) \subset V$, which proves the continuity of $\tau$. If we consider the operator $\Phi^{-1}: B \rightarrow A$ and the mapping $\tau^{-1}: \delta B \rightarrow \delta A$, the same arguments imply that $\tau^{-1}=\psi$ is also continuous, which completes the proof.

When applied to the operator $\Phi^{-1}: B \rightarrow A$ and the mapping $\tau^{-1}: \delta B \rightarrow \delta A$, Lemma11]implies that $|g(y)| \leq\left|\left(\Phi^{-1}(g)\right)\left(\tau^{-1}(y)\right)\right|$ for any $y \in \delta B$ and every $g \in B$. By letting $g=\Phi(f), f \in A$, and $y=\tau(x), x \in \delta A$, we obtain $|(\Phi(f))(\tau(x))| \leq$ $|f(x)|$. Together with Lemma 11 this inequality implies the following:

Corollary 2. If $\Phi: A \rightarrow B$ is a surjective, unital, and peripherally multiplicative operator, then $|(\Phi(f))(\tau(x))|=|f(x)|$ for any $x \in \delta A$ and every $f \in A$.

\section{The Main Theorem}

Here we prove the Main Theorem, stated in the first section. First we will show that, under its assumptions, equality (7) holds for every $x \in \delta A$ and any $f \in A$.

Proposition 2. If $\Phi: A \rightarrow B$ is a surjective, unital, and peripherally multiplicative operator between two uniform algebras, then for every generalized peak point $x \in \delta A$ the equality

$$
(\Phi(f))(\tau(x))=f(x)
$$

holds for all $f \in A$ and $x \in \delta A$.

Proof. By Lemma 9 the mapping $\tau$ from (6) is well defined, and according to (7), for a fixed $x \in \delta A$, all peaking functions $h \in \mathcal{F}_{x}(A)$ satisfy equality (9). Let $x_{0}$ be a generalized peak point of $A$, and let $f \in A$. Without loss of generality we can assume that $f\left(x_{0}\right) \neq 0$, since if $f\left(x_{0}\right)=0$, then also $(\Phi(f))\left(\tau\left(x_{0}\right)\right)=0$ by Lemma 10, if applied to the operator $\Phi^{-1}: B \rightarrow A$, the function $\Phi(f) \in B$ and the mapping $\tau^{-1}: \delta B \rightarrow \delta A$. Let $V$ be an open neighborhood of $x_{0}$. Bishop's Lemma implies that there is a peaking function $h \in \mathcal{F}_{x_{0}}(A)$ so that $P(h) \subset V$ and such that the function $(f h)(x)$ attains its maximum modulus within $P(h) \subset V$ exclusively. Let $z_{V}$ be a point in $\delta B$ so that $\left|(\Phi(f) \Phi(h))\left(z_{V}\right)\right|=\|\Phi(f) \Phi(h)\|$. Hence $(\Phi(f) \Phi(h))\left(z_{V}\right) \in \sigma_{\pi}(\Phi(f) \Phi(h))=\sigma_{\pi}(f h)$ by the peripheral multiplicativity of $\Phi$. Consequently, $(\Phi(f) \Phi(h))\left(z_{V}\right)=(f h)\left(\xi_{V}\right)$ for some $\xi_{V} \in X$. Then $\xi_{V} \in P(h)$ by the choice of $h$, since $\left|(f h)\left(\xi_{V}\right)\right|=\|f h\|$. Hence we have found points $z_{V} \in \delta B$ and $\xi_{V} \in P(h)$ so that

$$
f\left(\xi_{V}\right)=(f h)\left(\xi_{V}\right)=(\Phi(f) \Phi(h))\left(z_{V}\right) .
$$

The surjectivity of $\tau$ implies that $z_{V}=\tau\left(x_{V}\right)$ for some $x_{V} \in \delta A$. Equality (10), Corollary 2 and (7) imply

$$
\begin{aligned}
\left|f\left(\xi_{V}\right)\right| & =\left|(\Phi(f) \Phi(h))\left(z_{V}\right)\right|=\left|(\Phi(f))\left(\tau\left(x_{V}\right)\right)\right|\left|(\Phi(h))\left(\tau\left(x_{V}\right)\right)\right| \\
& =\left|f\left(x_{V}\right)\right|\left|h\left(x_{V}\right)\right|=\left|(f h)\left(x_{V}\right)\right| \leq\|f h\|=\left|f\left(\xi_{V}\right)\right| ;
\end{aligned}
$$

thus $\left|(f h)\left(x_{V}\right)\right|=\left|f\left(\xi_{V}\right)\right|=\|f h\|=\max _{\xi \in X}|(f h)(\xi)|$. Since this maximum is attained only within $P(h), x_{V} \in P(h)$, and according to (17), $(\Phi(h))\left(z_{V}\right)=$ $(\Phi(h))\left(\tau\left(x_{V}\right)\right)=h\left(x_{V}\right)=1$. Now equality (10) becomes $f\left(\xi_{V}\right)=(\Phi(f))\left(z_{V}\right)=$ $(\Phi(f))\left(\tau\left(x_{V}\right)\right)$. Therefore any neighborhood $V$ of $x_{0}$ contains points $\xi_{V}$ and $x_{V}$ such that $f\left(\xi_{V}\right)=(\Phi(f))\left(\tau\left(x_{V}\right)\right)$. The continuity of $f, \Phi(f)$, and $\tau$ yields $f\left(x_{0}\right)=$ $(\Phi(f))\left(\tau\left(x_{0}\right)\right)$. 
Note that since $\tau$ is a homeomorphism, we can rewrite (9) as $(\Phi(f))(y)=$ $f\left(\tau^{-1}(y)\right), y \in \delta B$. We see that, in fact, $\Phi$ is the composition operator on $A$ determined by $\tau^{-1}$.

The MAin Theorem. A surjective, unital, and peripherally multiplicative operator $\Phi: A \rightarrow B$ between two uniform algebras is an isometric algebra isomorphism.

Proof. Proposition 2 implies that the equality $(\Phi(f))(\tau(x))=f(x)$ holds for every $x \in \delta A$ and all $f \in A$. Therefore, the operator $\Phi^{\prime}:\left.\left.A\right|_{\delta A} \rightarrow B\right|_{\delta B}$ defined by $\Phi^{\prime}\left(\left.f\right|_{\delta A}\right)=\left.\Phi(f)\right|_{\delta B}, f \in A$, is an algebra isomorphism from $\left.A\right|_{\delta A}$ onto $\left.B\right|_{\delta B}$. Since the Choquet boundary of an algebra is in fact an algebra boundary, $\left.A\right|_{\delta A} \cong A$ and $\left.B\right|_{\delta B} \cong B$, while $\Phi$ is uniquely determined by $\Phi^{\prime}$. Consequently, $\Phi$ is an algebra isomorphism between $A$ and $B$.

\section{Non-Unital OPERATORS}

The assumption that $\Phi$ is unital is not too restrictive. In fact, if $\Phi$ is not unital, then, instead of being a composition operator as before, $\Phi$ is a weighted composition operator on $A$.

Proposition 3. Any surjective and peripherally multiplicative operator $\Phi: A \rightarrow B$ between two uniform algebras is the product of a function in $B$ and an algebra isomorphism from $A$ onto $B$. More precisely, there exists an isometric algebra isomorphism $\widetilde{\Phi}: A \rightarrow B$, such that

$$
\Phi(f)=\kappa \cdot \widetilde{\Phi}(f)
$$

for all $f \in A$, where $\kappa=\Phi(1) \in B$ and $\operatorname{Ran}(\kappa) \subset\{ \pm 1\}$.

Proof. Define $\widetilde{\Phi}: A \rightarrow B$ by $\widetilde{\Phi}(f)=\Phi(1) \cdot \Phi(f)$. Then $\widetilde{\Phi}$ is a surjective operator.

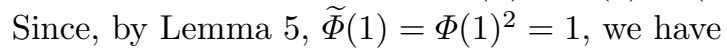

$$
\sigma_{\pi}(\widetilde{\Phi}(f) \widetilde{\Phi}(g))=\sigma_{\pi}\left(\Phi(1)^{2} \cdot \Phi(f) \Phi(g)\right)=\sigma_{\pi}(\Phi(f) \Phi(g))=\sigma_{\pi}(f g) .
$$

Thus $\widetilde{\Phi}$ is a surjective, unital, and peripherally multiplicative operator from $A$ onto $B$. By the Main Theorem, it is an isometric algebra isomorphism. This proves the proposition, since $\Phi(f)=\Phi(1)^{-1} \cdot \widetilde{\Phi}(f)=\Phi(1) \cdot \widetilde{\Phi}(f)$. The inclusion $\operatorname{Ran}(\kappa) \subset\{ \pm 1\}$ follows from Lemma 5 ,

If $Y$ is connected, then $\kappa \equiv 1$ or $\kappa \equiv-1$. Therefore, in this case, either $\Phi$ or its negative is an isometric algebra isomorphism.

Let $X=\mathcal{M}_{A}$ and $Y=\mathcal{M}_{B}$. Since $\widetilde{\Phi}$ is an isometric algebra isomorphism, there exists a homeomorphism $\psi: Y \rightarrow X$ such that $(\widetilde{\Phi}(f))(y)=f(\psi(y))$ for all $f \in A$ and all $y \in Y$. Hence,

$$
(\Phi(f))(y)=(\Phi(1))(y) \cdot(\widetilde{\Phi}(f))(y)=(\Phi(1))(y) \cdot f(\psi(y)) .
$$

We have obtained the following:

Corollary 3. Let $A$ and $B$ be uniform algebras on their maximal ideal spaces $X$ and $Y$, respectively. If $\Phi: A \rightarrow B$ is a surjective and peripherally multiplicative operator, then there exists a homeomorphism $\psi: Y \rightarrow X$, such that

$$
(\Phi(f))(y)=\kappa(y) \cdot f(\psi(y))
$$

for all $f \in A$ and all $y \in Y$, where $\kappa=\Phi(1)$ is as in Proposition 3. In particular, $\Phi$ is automatically a linear operator. 
Since every multiplicative operator that preserves the peripheral ranges of algebra elements is peripherally multiplicative, we obtain the following:

Proposition 4. Any surjective and multiplicative operator $\Phi$ between two uniform algebras that preserves the peripheral ranges of algebra elements is of the form (11).

If, in addition, $\Phi$ is unital, then it is an algebra isomorphism. Namely,

Corollary 4. Any surjective, unital, and multiplicative operator between two uniform algebras that preserves the peripheral ranges of algebra elements is an isometric algebra isomorphism.

Note that any operator $\Phi: A \rightarrow B$ that preserves the spectra or the ranges of algebra elements preserves also their peripheral spectra and is unital. Consequently,

Corollary 5. Any surjective and multiplicative operator between two uniform algebras that preserves the spectra or the ranges of algebra elements is an isometric algebra isomorphism.

Observe that elements with equal spectra or ranges have equal peripheral spectra. Therefore, Corollary 3 implies the results obtained by N. V. Rao and A. K. Roy in [9], and by Hatori, Miura, and Takagi in [4. Namely,

Corollary 6. Any surjective operator $\Phi: A \rightarrow B$ between two uniform algebras that satisfies the spectral multiplicativity condition $\sigma(\Phi(f) \Phi(g))=\sigma(f g)$, or, the range-multiplicativity condition $\operatorname{Ran}(\Phi(f) \Phi(g))=\operatorname{Ran}(f g)$ for all $f, g \in A$, is of the form (11).

\section{REFERENCES}

[1] A. Browder, Introduction to Function Algebras, W. A. Benjamin, Inc., 1969. MR0246125 $(39: 7431)$

[2] S. Grigoryan and T. Tonev, Shift-Invariant Uniform Algebras on Groups, Monografie Matematyczne 68, New Series, Birkhäuser Verlag, Basel-Boston-Berlin, 2006. MR2239563

[3] K. Jarosz, When is a linear functional multiplicative?, Contemporary Math., 232(1999), 201210. MR:1678332 (2000b:46085)

[4] O. Hatori, T. Miura and H. Takagi, Characterizations of isometric isomorphisms between uniform algebras via nonlinear range-preserving properties, Proc. Amer. Math. Soc., 134(2006), 2923-2930. MR 2231616

[5] O. Hatori, Private communication, 2006.

[6] S. Kowalski and Z. Słodkowski, A characterization of maximal ideals in commutative Banach algebras, Studia Math., 67(1980), 215-223. MR592387(82d:46070)

[7] G. Leibowitz, Lectures on Complex Function Algebras, Scott, Foresman and Co., 1970. MR0428042 (55:1072)

[8] L. Molnár, Some characterizations of the automophisms of $B(H)$ and $C(X)$, Proc. Amer. Math. Soc. 130(2005), 1-11. MR1855627 (2002m:47047)

[9] N. V. Rao and A. K. Roy, Multiplicatively spectrum-preserving maps of function algebras, Proc. Amer. Math. Soc. 133(2005), 1135-1142. MR2117215 (2005k:46124)

[10] N. V. Rao, T. V. Tonev and E. T. Toneva, Uniform Algebra Isomorphisms and peripheral spectra, Contemporary. Math., 427 (2007), 401-416.

[11] W. Żelazko, Banach Algebras, Elsevier Publ. Co., 1973. MR0448079 (56:6389)

Division of Science and Mathematics, Bethany Lutheran College, Mankato, MinNESOTA 56001

E-mail address: luttman@blc.edu

Department of Mathematical Sciences, The University of Montana/Missoula, MonTANA 59812-1032

E-mail address: tonevtv@mso.umt.edu 